\title{
Intrinsic Limitations of the Human Mind
}

\author{
Atanu Bikash Chatterjee \\ Indian Astrobiology Research Centre, Mumbai \\ India \\ E-mail: achatterjee.jur@gmail.com
}

\begin{abstract}
"Can machines think?" this was a question proposed by Alan Turing about half a century ago. The significance of that single question gave birth to the extremely interesting field of Artificial Intelligence. The computational resources have since grown and I believe are growing exponentially. Our understanding of the nature and its mechanisms has increased vastly. All branches of science that were earlier thought to be separate entities and self - contained in their own domains are merging incessantly. However, everything that exists in this universe is constrained to certain inherent limitations. These limitations are based on the fundamental constants which are governing the cosmos. Based on these limitations, Seth Lloyd attempted to formulate the finite limitation of computational processes. The human brain is a complex, adaptive and a self-organizing system. Intelligence is an emergent property of this complex networked structure but, this intelligence comes at cost of gradual dissipation of information (entropy generation). Thus, the human brain is a dissipative system, an entropy producing machine like, a computer that constantly generates entropy through the cognitive processes occurring within its intricately fractalized networked structure. The intrinsic limitation to our thought processes, the computing power of our mind is thus investigated in this paper.
\end{abstract}

Keywords: Artificial Intelligence, cognitive process, complex systems, entropy, organization, self-organization.

\section{Introduction}

Computers are devices that can be programmed to carry out a finite set of logical and arithmetical operations. What it can do or, it cannot is clearly defined by us 
through a set of algorithms. Even though, the computational power has increased and continues to increase exponentially over the years as predicted by Gordon Moore, the Intel co-founder in 1970 [1], the dissipation of energy limits its computational power and manipulating capabilities [2]. Generally, all processes in nature are constrained due to dissipative effects. It is the dissipative effects present in natural systems that make natural processes irreversible. Thermodynamically, irreversible processes generate entropy. Entropy is an inferior grade of energy [3]. Practically, all processes generate entropy from natural to artificial. The versatile software packages that are used to simulate complicated phenomena, statistical data etc. generate entropy [4]. Computational processes are thus, irreversible. The high-speed processors, RAMs and GPUs that we use today degrade almost, all the energy supplied to them into heat (a lowgrade form of energy) hence, generating huge amount of entropy [5]. Thus, we have been constantly generating entropy through technological revolutions and converging (the human race) towards a situation of "heat death" when all matter and radiation will be equilibrated, as postulated by the Second Law of Thermodynamics [6,7]. Undoubtedly, entropy will increase with even a miniscule activity performed anywhere in the world. Shannon [8] related entropy to loss of information and Lambert [9] related entropy to dispersal of energy from a confined region to free space. Different interpretations of entropy thus, point towards a global conclusion that, entropy is the loss or degradation of orderliness in nature or (of) natural systems. But, natural systems don't seem to follow the entropy principle [10]. They become organized with time and evolve into much more complicated species. They adapt [11], organize, optimize [12, 13] and selforganize $[14,15,16,17,18,19,20]$ with time. Through continuous selforganizing processes these systems prevent themselves from degrading although, some entropy is always generated [21]. Through this paper, we argue on the basis of the rate of self-organization the ability of a natural system to compute and the ability of these systems to minimize entropy generation through efficiently operating feed-back loops.

\section{Human Brain as a Self - Organizing Entity}

The brain forms the center of the nervous system of all vertebrates and most invertebrate animals. It is the sole decision making entity and the most complex organ of the entire body. From philosophical perspective, what makes the brain different from rest of the body organs is that, it generates the mind. Mind and matter were thought to be separate entities. The mind-body problem or the mindbody dualism was pointed out and formulated in the present form by the French philosopher and mathematician, René Descartes [22]. However, today we have two different schools of philosophy namely, Dualism and Monism. According to Monism, mind and body are not two physiologically and ontologically different entities [23]. Mind is the complex of all cognitive processes - consciousness, 
reasoning, perception and judgment. Various approaches have since, been devised to understand the functioning of the intricately complex brain. Traditionally, Neural Network theory has been viewed as a simplified version of neural processing processes in brains. The idea of Artificial Neurons, to imitate the functioning of the human brain was first proposed by Warren McCulloch, a neurophysiologist and Walter Pitts, a logician in 1934 [24]. The human brain can be modeled as a complex networked structure with multiple nodes and vertices connecting the neurons. Every complex system shows a distinctive property of emergence $[25,26]$. It is this property that separates a complex system from a simple system. What could be the emergent property for the human brain? It is the human Intelligence. The human race has evolved with time and has continuously adapted itself to the ever-changing environment through the various selforganizing processes. The memory and computational power of the brain has increased immensely from primates to Homo sapiens. The entropy generating rate has also increased but, not at the same rate. It is due to the self-organizing processes and efficient feed-back loops operating between the surrounding environment and the neural processes that, the entropy generation has been minimized and information sustainability and storage capacity has increased [20]. Thus, we can directly relate the rate of self-organization to the efficiency of various cognitive processes occurring within the brain. Thus, it is the rate of selforganization that, limits the human thought processes.

\section{Limitation of the Cognitive Processes}

In his paper, "Ultimate physical limits to computation", Seth Lloyd investigates the physical limitations on computation and relates it with the fundamental physical constants, $G, \hbar$ and $c$ [2]. The total number of logic operations performed per second is equal to the sum over all logic gates of the operations per second per gate. The logical operations performed per second is given by him as per the equation,

$\sum_{l} \frac{1}{\Delta t_{l}} \leq \sum_{l} \frac{2 E_{l}}{\pi \hbar}=\frac{2 E}{\pi \hbar}$

based, on the interpretation of the evolution of an orthogonal (distinguishable) quantum state that, a quantum state with spread in energy $\Delta E$ takes rather a time, $\Delta t$, where,

$\Delta t=\frac{\pi \hbar}{2 \Delta E}$ to evolve into an orthogonal state.

Based on above equations, the amount of organization can be defined in a networked complex system [27]. The amount of organization present in a system is inversely proportional to the average sum of all actions of its constituting 
elements. Thus, we can quantitatively define organization as the number of quanta dispersed per unit motion per unit inter-nodal displacement.

$\operatorname{organization}(\alpha)=\frac{n m \hbar}{\sum_{i=0}^{n} \sum_{j=0}^{m} I_{i j}}=\frac{n m \hbar}{\sum_{i=0}^{n} \sum_{j=0}^{m} \int_{t_{1}}^{t_{2}} L_{i j} d t}$

In the above equation, $I_{i j}$ and $L_{i j}$ represent the action and Lagrangian respectively for $i^{\text {th }}$ element for the $j^{\text {th }}$ inter-nodal displacement. The rate of self-organization can be defined as the time derivative of the organization. The loop will be generated due to the non-linearity of the inter-elemental interaction. The closure or loop [28] would be due the peculiarity of non-linear equations, the stretching and folding nature of them [25]. The emission of quanta from dissipative systems in nature has a very profound importance. The ultimate speed of information transmission is constrained the velocity of light in vacuum or celeritas $(c)$. Organization will disperse energy and hence, generate entropy. The dispersed energy and information from the system is transmitted in form of quanta. An observer receives the information (in form of quanta) and interprets the phenomena from the immediately acquired information. The emergent intelligence of the observer will allow him to develop an understanding of the phenomena as per his perspective. Thus, the scientific depths of all natural phenomena are relative [21]. Thus, the inherent limitations to our perception and understanding of any phenomena is directly related to the efficiency or the rate of self-organizing processes that, aim towards greater fractalization of the neural circuitry, reduced entropy generation and accelerated parallel neural computation.

\section{Conclusion}

The aim of this paper is to develop an insight into the mind-body problem (duality) and to define mind as a complex emergent property, a qualitative aspect of the complex neural circuitry of the human brain. The brain which was earlier thought to be 'something unusual' has been reduced to a simple entropy producing machine like all computational devices. The one part where the brain differs from all artificial machines is in its ability to adapt with its surroundings through continuous self-organizing processes and feedback loops. The rate of selforganization governs the computational power of the brain and also the complex cognitive processes. The limitation thus imposed is intrinsic and not affected by the surrounding. This limitation to human thought process presents a paradox, "Are all physical laws relative?" Here, the 'relative' implies the relative rates of self-organization processes. 


\section{References}

[1] Moore, G.E., "Cramming more components onto integrated circuits", Electronics Magazine, Volume 38 (8), (1965).

[2] Lloyd, Seth, "Ultimate physical limits to computation", Nature 406, (2000), pp. 1047 - 1054.

[3] Nag, P.K., Engineering Thermodynamics, Tata McGraw Hill Publications, New Delhi (2008).

[4] Vidal Clément, "The Future of Scientific Simulations: from Artificial Life to Artificial Cosmogenesis", In Death and Anti-death, Thirty Years After Kurt Gödel, Ria University Press (2008).

[5] Anandan, S.S. and Ramalingam V., "Thermal Management of Electronics: a review of literature", Thermal Science, Volume 12 (2), (2008).

[6] Hawking, S.W., "Black holes and thermodynamics", Physical Review D, Volume 13 (2), (1976).

[7] Thomson, W., "On the universal tendency in nature to the dissipation of mechanical energy", Proceedings of the Royal Society of Edinburgh, Volume 1 (59), (1852).

[8] Shannon, C., "A mathematical theory of communication", Bell System Technical Journal, Volume 28 (4), (1948).

[9] http://www.2ndlaw.oxy.edu/, retrieved on $23^{\text {rd }}$ June, 2012.

[10] Prigogine, I., Introduction to the Thermodynamics of Irreversible Processes, Wiley, (1961).

[11] Bar-Yam, Y., Dynamics of Complex Systems, Addison Wesley, USA, (1997).

[12] Bejan, A., Advanced Engineering Thermodynamics, John Wiley, USA, (1998).

[13] Bejan, A., Design in Nature, Random House Inc., UK, (2012).

[14] Chatterjee, A. B., "Certain Interesting Properties of Action and its Application Towards Achieving Greater Organization in Complex Systems", Complexity Digest, Volume 23 (11), (2011).

[15] Chatterjee, A. B., "Principle of Least Action and Theory of Cyclic Evolution", Complexity Digest, Volume 23 (11), (2011).

[16] Chatterjee, A. B., "Game - theoretic formulation of complex systems", Sixth International Conference on Game Theory and Management, (2012), (accepted).

[17] Chatterjee, A. B., "Emergence of structures and forms in complex adaptive systems in nature", Sixth International Conference on Relating Design in Nature with Engineering and Science, (2012), (accepted).

[18] Georgiev, G. and Georgiev, I., "The Least Action and the Metric of an Organized system”, Open Systems and Information Dynamics, Volume 9(4), (2002).

[19] Chow et al, "Nonlinear pedagogy: Learning design for self-organizing neurobiological systems", New Ideas in Psychology, Volume 29, (2011), pp. $189-200$. 
[20] Chaisson, E. J., Order, Form and Complexity, Harvard University Press, Cambridge, (1998).

[21] Chatterjee, A. B., "Interactions of Self-organizing Systems in Nature", Cogprints, (2012).

[22] Descartes, R., "Meditations on First Philosophy", (1641). The Philosophical Writings of René Descartes, Cambridge University Press, (1984).

[23] http://www.plato.stanford.edu/, retrieved on $26^{\text {th }}$ June, (2012).

[24] McCulloch W. and Pitts W., "A Logical Calculus of Ideas Immanent in Nervous Activities", Bulletin of Mathematical Biophysics, Volume 5 (4), (1943).

[25] Baranger, M., "Chaos, Complexity and Entropy: A physics talk for nonphysicist", New England Complex System Institute (NECSI), Massachusetts, USA, (2001).

[26] Georgiev, G., "A quantitative measure, mechanism and attractor for selforganization in networked complex systems", Sixth International Workshop on Self-Organizing Systems, (2012), (accepted).

[27] Akkerhuis, op Jagers Gerard, The Operator Hierarchy, Alterra Scientific Publications, The Netherlands, (2010). 